AIAA-2003-2116

\title{
DEVELOPMENT OF CONTROL ALGORITHM FOR THE AUTONOMOUS GLIDING DELIVERY SYSTEM
}

\author{
Isaac I. Kaminer ${ }^{\dagger}$ and Oleg A. Yakimenko \\ Department of Aeronautics and Astronautics, Naval Postgraduate School, Monterey, CA
}

\begin{abstract}
$\underline{\text { Abstract }}$
The paper considers the development and simulation testing of the control algorithms for an autonomous high-glide aerial delivery system, which consists of 650 sq.ft rectangular double-skin parafoil and $500 \mathrm{lb}$ payload. The paper applies optimal control analysis to the real-time trajectory generation. Resulting guidance and control system includes tracking this reference trajectory using a nonlinear tracking controller. The paper presents the description of the algorithm along with simulation results and ends with guidelines for the further implementation of the developed software aboard the real system.
\end{abstract}

\section{Introduction}

Maneuverable ram-air parafoils ${ }^{1-3}$ are widely used today. The list of users includes skydivers, smoke jumpers and special forces. Furthermore, their extended range (compared to that of round canopy parachutes) makes them very practical for payload delivery. Recent introduction of the Global Positioning System (GPS) made the development of fully autonomous ram-air parafoils possible. Moreover, ram-air parafoils are nowadays considered a vital element of the unmanned air vehicles (UAVs) capability and of International Space Station (ISS) crew rescue vehicle. ${ }^{4-12}$

Autonomous parafoil capability implies delivering the system to a desired landing point from an arbitrary release point using onboard computer, sensors and actuators. This requires development of a guidance, navigation and control (GNC) system. The navigation subsystem manages data acquisition, processes sensor data and provides guidance and control subsystems with information about parafoil states. Using this information along with other available system data (such as local wind profiles), the guidance subsystem plans the mission and generates feasible (physically realizable and mission compatible) trajectory that takes the para-

This paper is declared a work of the U.S. Government and is not subject to copyright protection in the United States.

\footnotetext{
${ }^{\dagger}$ Associate Professor; Senior Member AIAA.

${ }^{\dagger}$ Research Associate Professor, Associate Fellow AIAA.
}

foil from the initial position to the desired landing point. Finally, it is the responsibility of the control system to track this trajectory using the information provided by the navigation subsystem and onboard actuators.

In the past decade, several GNC concepts for gliding parachute applications have been developed and published. ${ }^{13-22}$ Most of them were tested in a simulation environment, some in flight test.

Some of the published papers include analytical development of feasible trajectories. In Ref.23, for example, minimum glide angle and descent rate, minimum turn radius and maximum gliding turn rate, reachable ground area, minimum altitude loss problems were considered and solved analytically for a simplified three-degree-of-freedom (3DoF) model with a parabolic lift-drag polar. The problems of the maximum time to stall the glider at the predefined point in a horizontal plane and a maximum range for the appropriate simplified model with constraints were also considered. Optimization included no wind scenario.

Assuming the wind velocity to be constant Ref.24 synthesized zero-lag control of the gliding parachute system with the constant rate of descent. This interesting work uses a compound performance index blending time, kinetic energy, and controls expenditure. The optimal problem was solved by applying Pontrjagin's maximum principle. ${ }^{25}$ The resulting two-point boundary-value problem was solved using KrylovChernous'ko method. ${ }^{26}$

However, majority of the published results on GNC system development for parafoils sue heuristic rules and classical control algorithms. It is appropriate to mention some of them here emphasizing a scale of the system, sensor suite specs and control approach.

Probably one of the first GNC algorithms for small ram-air parachutes is presented in Ref.13. Computer programs were written using $6 \mathrm{DoF}$ models of small parafoils, such as Ram-air, Para-commander, and Standard Round. The aerodynamic models were simplified, but they included the non-linearities and severe constraints common to all parachutes as compared to aircraft. The algorithms used GPS position and velocity data provided at $1 \mathrm{~Hz}$ to control both toggle lines. Homing and circling algorithms were simulated using direc- 
tion sines and cosines. Wind was included in all algorithms. Homing algorithms were included that increased the allowable offset, minimized the miss distance and reduced the impact velocity. These were based on two-axis proportional navigation (PN), and also upon upward-biased rate-integrating navigation in glide angle. The latest produced final flare and nearly vertical landing even from a long initial offset. For the case of high initial altitude and small offset circling algorithms were also introduced. Finally, combined downwind, upwind, and guidance algorithms were simulated to emulate manned parachute procedures.

Another approach was employed in Ref.16 where Pseudo-Jumper (PJ) guidance and control algorithms based on the professional jump procedures were introduced. The main phases of the mission after release were defined as follows:

$\checkmark$ "Cruise" phase consisting of aiming for the holding area way-points (WPs) by one or more types of homing algorithms;

$\checkmark$ "Hold" phase: As the parachute gets close enough to the homing WPs, it is caused to perform S-turns just downwind of the approach zone. The pattern is adjusted to suit the wind speed so that it stays near the landing zone (LZ) and has just enough upwind distance left for the approach and flare;

$\checkmark$ "Center" phase: at some point, governed mainly by altitude (time-to-go), the PJ must aim for the center of the drop zone so that it is heading towards the touchdown target. Enough time must be allowed to complete any ongoing S-turn;

$\checkmark$ "Approach" phase: at some point, the PJ has to stop trying to center and get lined up with the surface wind direction and to minimize toggle actions past that point;

$\checkmark$ "Flare" phase is triggered with rapid full braking to arrest the vertical and/or horizontal inertial velocities in an optimal control fashion.

Perfect knowledge of parafoil's relative location, i.e. availability of a GPS sensor and of sonic altimeter as well as of winds below $300 \mathrm{ft}$ was assumed.

Recent work by the Iowa State University (http://cosmos.ssol.iastate.edu/RGS) ${ }^{27,28}$ and Atair Aerospace (http://www.extremefly.com/aerospace) includes development of a parafoil-based Recovery Guidance System to return payloads from a highaltitude balloon back to the ground safely and predictably. The parafoil is supposed to accompany the balloon and to be cut free of the balloon at a predetermined time. It then guides the payload to a designated LZ. As reported, fully automated guidance hardware and software used DGPS (differential GPS) data, a digital compass, a linear quadratic regulator and $\mathrm{PN}$ guidance to control and navigate the parafoil. Only simulation results have been reported so far.
Another GNC system incorporating appropriate data acquisition system was developed to support highaltitude-high-opening insertion into hostile environments and was supposed to be used with a $500 \mathrm{lb}$ parafoil. The GPS guidance mode was designed to steer a glider to the LZ using only GPS data or GPS plus up to eight additional sensors. The GPS data was provided at $1 \mathrm{~Hz}$ and inertial sensors at $200 \mathrm{~Hz}$. Control steering was accomplished by actuating one of the two steering controls, which allowed four flight modes: straight flight, steer left or right, hard turn left or right, and flare. No test results however are available so far.

Another project was reported by the German Aerospace Center. It aimed to identify behavior of a small $(200 \mathrm{lbs})$ and extensively instrumented parafoil-based system and to investigate GNC-concepts for the autonomous landing. ${ }^{29,30}$ Three and four degree of freedom models were developed and parameters of these models were identified during this study.

The world's largest parafoil was designed for the U.S. Army by Pioneer Aerospace Corp. This was a part of a contract undertaken by Pioneer and the U.S. Army Soldier Systems Command and was tested in 20012002 at the U.S. Yuma Proving Grounds, AZ (YPG) as an aid for ISS "lifeboat". The resulting parafoil was ultimately intended to evacuate a seven-person crew from the station in case of an emergency (Fig.1). ${ }^{11-12}$

The $1,600 \mathrm{lb}$ (50 times the weight of a typical personal parachute) 7,500sq.ft parafoil wing has the wingspan wider than a football field and a canopy area of more than one and a half times that of the wings of a 747 jumbo jet and is designed to carry weights of up to $42,000 \mathrm{lbs}$, the heaviest cargo ever transported by a parafoil. The system is able to move anything from supplies to armored vehicles into strategic areas. Other applications could include mentioned recovery of highvalue assets for the space program, and humanitarian aid, such as the delivery of food, supplies and medications to war-torn areas like Bosnia, Sarajevo, Afghanistan, and Iraq.

The Pioneer Aerospace Corporation is an industry leader in parachute design. They have provided recovery parachutes for NASA's space shuttle, the Air Force's B-1A Bomber and parachutes for other NASA and Department of Defense programs. Since testing on the Guided Parafoil Aerial Delivery System (GPADS) program began in January 1994, Pioneer holds records for the largest parafoil, highest altitude $(22,000 \mathrm{ft})$, and heaviest weight $(15,500 \mathrm{lbs})$ airdropped.

The GNC system for GPADS ${ }^{7,8}$ was developed by the European Space Agency. ${ }^{22,29-33}$ The Parafoil Technology Demonstrator program was set up and conducted under ESA to demonstrate feasibility of guiding a large-scale parafoil autonomously to a predefined point and to perform a flared landing within a specific range. Another objective was to enable further investi- 
gation into the flight dynamics of the parafoil systems. The first automatically-guided, parafoil based descent and landing of a test vehicle was carried out in support of ESA's development in 1997. The spacecraft was supposed to be completely automated, although the crew will have the capability to switch to backup systems and steer the parafoil manually, if necessary.
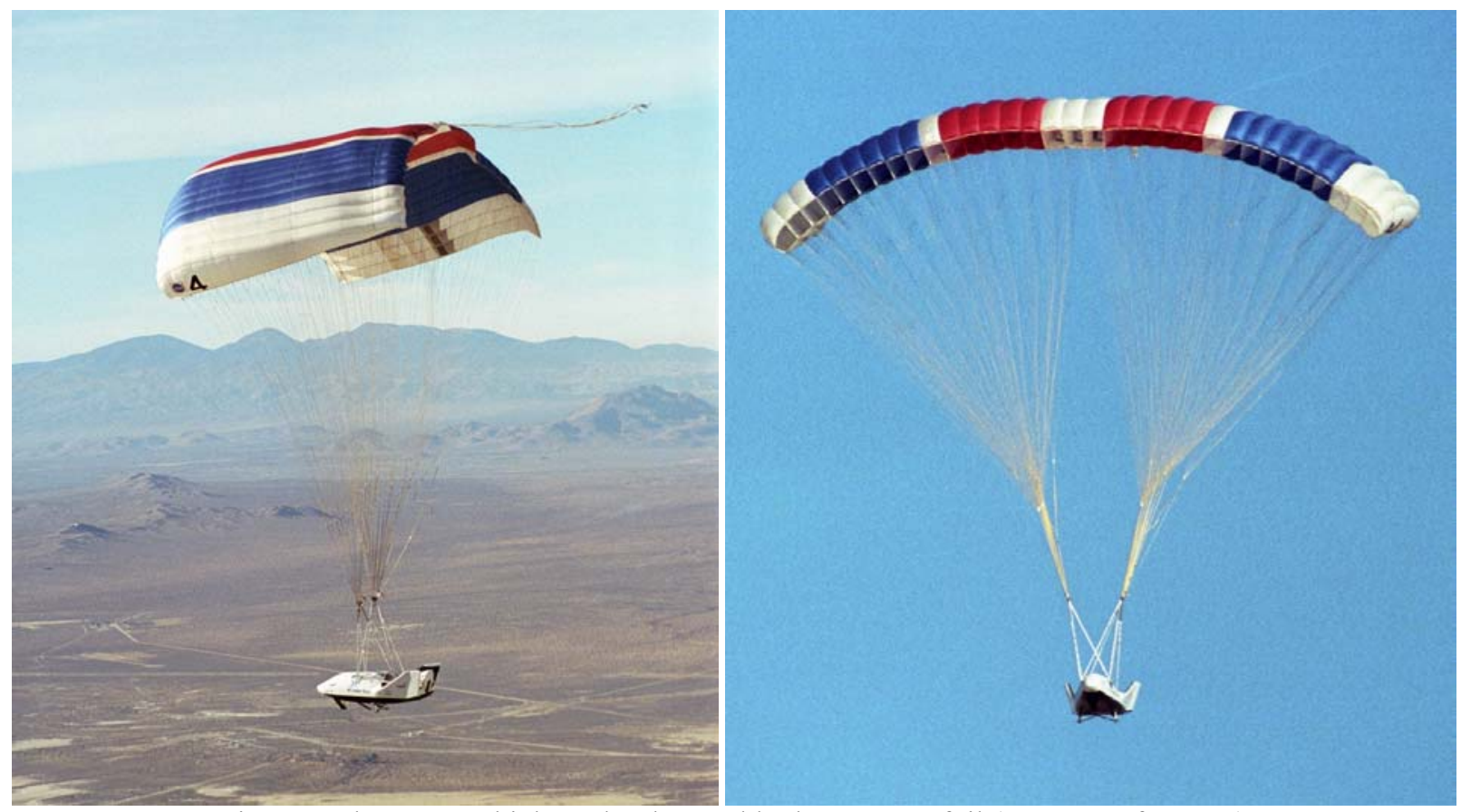

Figure 1 The X-38 vehicle under the world's largest parafoil (courtesy of NASA)

The prototype reference trajectory (RT) consisted of the following nominal phases

$\checkmark$ Acquisition - trajectory initialization and initial turn;

$\checkmark$ Homing - travel to intercept Energy Management Circle (EMC);

$\checkmark$ EMC Entry, EMC and EMC Exit - the energy management circling maneuver;

$\checkmark$ Final - final approach to target on desired heading;

The GNC system employs laser or radar altimeter to trigger flare and is required to steer the parafoil to within $100 \mathrm{~m}$ of a preselected desired impact point (DIP) (so far the accuracy of $200 \mathrm{~m}$ was achieved on a smaller scaled prototypes).

To support the development of GPADS software and to evaluate its expected performance on ram-air parafoils with payload capacities ranging from less than $200 \mathrm{lbs}$ to $40,000 \mathrm{lbs}$, Draper Laboratory also developed a GNC package. ${ }^{17-19}$ This software was demonstrated using NASA Dryden Flight Research Center airdrop test article known as the "Wedge 3" which utilizes an 88sq. ft ram-air parafoil and carries a payload of $175 \mathrm{lbs}$. Same GNC algorithms were also tested in simulation using the model of a larger 3,600sq.ft guided parafoil air delivery system with a $13,088 \mathrm{lb}$ payload. Upon full deployment of the parafoil canopy the GNC algorithm is activated. It determines the desired heading angle that will lead to a touchdown at LZ. Seven guidance modes are available:

$\checkmark$ Automatic trim;

$\checkmark \quad$ Maneuver to WP;

$\checkmark$ Transition to hold;

$\checkmark$ Holding pattern (to maintain turn radius);

$\checkmark$ Maneuver to target;

$\checkmark \quad$ Landing approach;

$\checkmark \quad$ Landing flare.

The control system may switch between any of these modes sequentially or bypass some of them.

The guidance concept presented in Ref.22 follows generally the same scheme. The energy management concept is adopted motivated by the way novice skydivers learn the landing approach. ${ }^{16}$ The trajectory is planned using WPs. The guidance works as follows:

$\checkmark$ Homing: from the current position the energy management center (EMC) is approached if possible. If the remaining distance is not long enough to reach the desired impact point, the WP is moved in the direction to the final turn point (FTP);

$\checkmark$ Energy Management: after reaching EMC, surplus altitude is reduced by flying S-patters to the energy management turn points (EMTP). If the remaining distance becomes too small to reach DIP, the EMTP is shifted along the energy management axis towards the EMC; 
$\checkmark$ Landing: the landing phase is divided into 4 subphases: (1) transition into landing corridor, (2) approach of final turn point (FTP), (3) turn into wind and (4) flare.

The Backup mode was also developed as follows: if altitude and subsequently remaining distance are too small to reach the landing point during homing phase, the vehicle keeps heading the FTP until a specified decision altitude is reached. Then the vehicle turns against wind independently of its position and prepares for landing.

The guidance system output consists of a heading command computed using current position and next WP. For wind accommodation, the trajectory is planned in the wind fixed coordinate system. ${ }^{21}$ Assuming constant horizontal wind, the actual position is shifted to a virtual position by the predicted wind drift, the vehicle will be affected during the remaining flight until touch down. Within this wind fixed system the guidance can be done as if there is no wind. Depending on the sign of the heading error, either the left or the right actuator is activated, leading to a left or a right turn. A saturation limited PN controller computes the desired actuator position. For instance, with an amplification of 4 , a commanded turn of $90^{\circ}$ first activates one actuator to full stroke until the heading difference goes below $15^{\circ}$ and the proportional part of the controller takes over.

According to Ref.22 after selective availability has been switched off (in May 2000), plain GPS data without differential corrections is accurate enough for trajectory planning. The GPS altitude, with errors of about $\pm 20 m$, is combined with the pressure altitude. Heading information is supposed to be computed by applying a complementary filter to blend azimuth and yaw rate as described in Refs.14-15. Simulation results also show that possible wind mispredictions have more influence on the landing accuracy, than incorrect estimates of model parameters and measurement errors. However, as long as the errors stay in the normal range (position $\pm 10 \mathrm{~m}$, altitude $\pm 5 \mathrm{~m}$, heading $\pm 10^{\circ}$ ) the GNC algorithm is robust enough to land the vehicle within a range of $\pm 50 \mathrm{~m}$ around the predefined landing point, provided that the wind is perfectly known.

As seen above in most GNC concepts, the guidance itself is divided into three phases: homing, energy management and landing, where each of them can consist of further subphases. Homing, i.e. flying towards the LZ, and landing are similar in almost all guidance concepts. Energy management is done by flying a certain pattern close to the landing point, until some criteria is satisfied, switching to the landing phase. Most differences among the realized guidance concepts are concerned with the shape of the pattern and the criteria for the landing decision.

Present paper addresses the problem of GNC development for a parafoil system as follows. First, a fea- sible trajectory is generated in real-time that takes the parafoil from initial release point to touch down at a desired impact point. It is assumed that only the direction of the wind at LZ is known. The trajectory consists of an initial glide, spiral descent and of final glide and flare. The final glide and flare are directed into the wind at LZ. This structure is motivated by optimal control analysis carried out on a simplified model. The resulting trajectory is tracked using a nonlinear algorithm with guaranteed local stability and performance properties (see Ref.34). The control algorithm converts trajectory-tracking errors directly into control actuator commands. Therefore, only GPS position and velocity are needed to implement it.

As expected the basic trajectory structure is similar to the ones reported in the literature. However, as shown in Section III, a single smooth inertial trajectory is generated using simple optimization and is tracked throughout the drop by the same control algorithm. This eliminates the need for multiple modes, extensive switching logic and wind information throughout the drop. The latter is made possible by the nonlinear control algorithm that tracks the inertial trajectory directly and treats wind as a disturbance.

This specific delivery system considered in this paper is called Pegasus. It consists of 650sq. ft span rectangular double-skin parafoil and 500 lb payload (Fig.2) and was originally manufactured by the FXC Corp. It can be controlled by symmetric and differential flap deflections that occupy outer four (out of eight on each side) cells of the parafoil.

This paper is organized as follows. Section II contains a brief description of the system and of the complete 6DoF model used to support the development of the GNC algorithm. Section III introduces optimal control strategy for a simplified parafoil model using Pontrjagin's principle of optimality. Section IV discusses real-time trajectory generation for the control algorithm. Section V discusses the development of the tracking control algorithm. Section VI introduces simulation results of the complete guidance and control system. Finally, Section VII contains the main conclusions.

\section{System Architecture and Modeling}

To support the development of the GNC algorithm, a complete 6DoF model of Pegasus was developed and tuned using flight test data provided by the YPG. Fig.3 outlines Pegasus dimensions.

The description of the $6 \mathrm{DoF}$ model used is given in Ref.35. This model matches flight test data and is characterized by the following integral parameters: the average descent rate is $3.7-3.9 \mathrm{~m} / \mathrm{s}$, glide ratio is about 3.0 , the turn rate of $\sim 6^{\circ} / \mathrm{s}$ corresponds to the full deflection of one flap. An interesting feature from the control standpoint is that the system exhibits almost no flare capability (flaps deflection results in almost no change 
in the descent rate). Therefore, it is very important to land it into the wind.

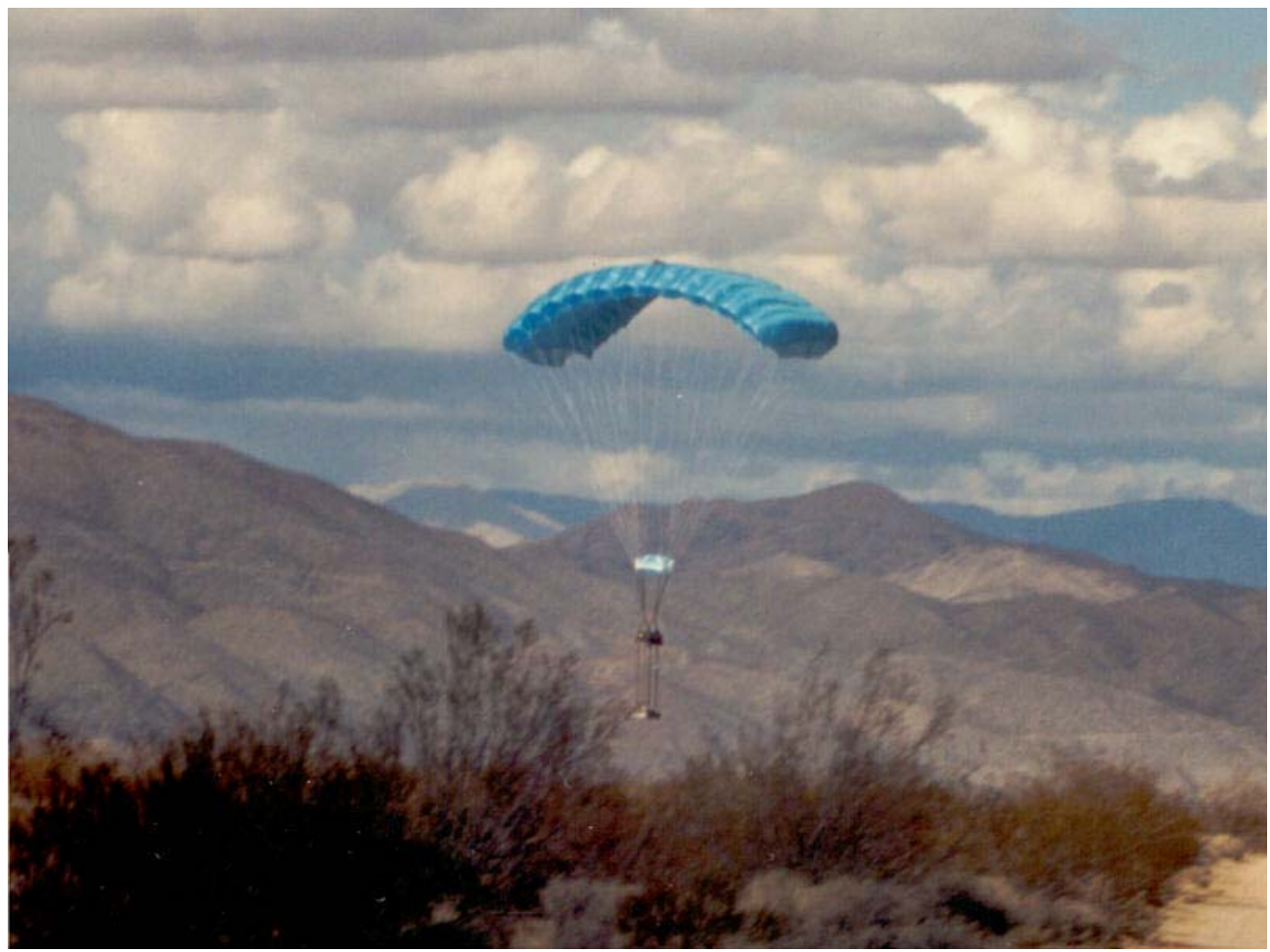

Figure 2 Double-skin 650sq.ft parafoil with 500lb payload
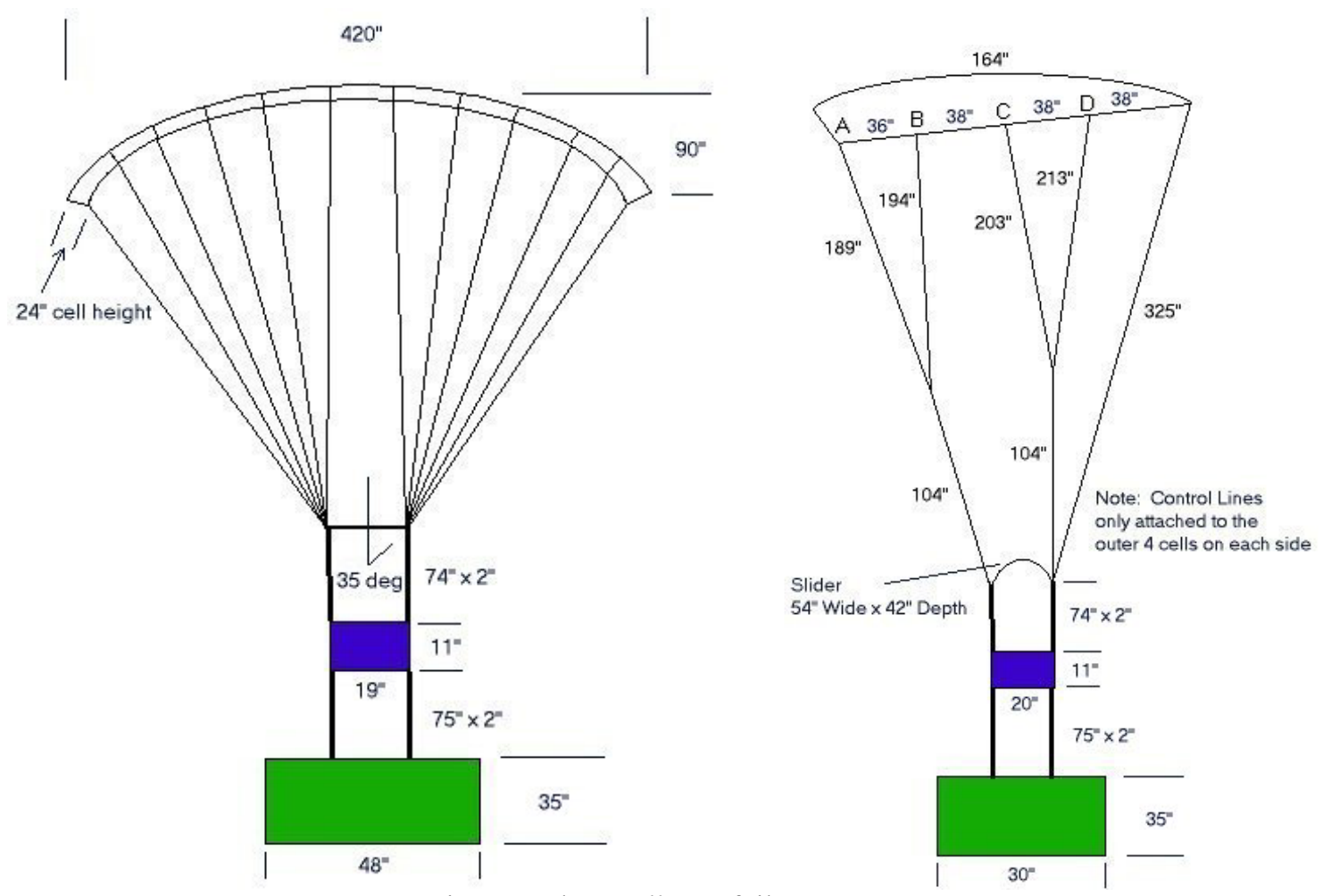

Figure 3 The $500 l b$ parafoil geometry 


\section{Optimal Control Synthesis}

Consider the following kinematic model of a parafoil in the horizontal plane. Suppose we have a constant glide ratio and by pulling risers we can control its yaw rate. Mathematically, this is expressed by the following simplified equations:

$$
\dot{x}=V \cos \psi, \dot{y}=V \sin \psi, \dot{\psi}=v,
$$

where $v \in[-\Xi ; \Xi]$ is the only control.

The Hamiltonian for the system (1) for a timeoptimal control can now be written as:

$$
H=p_{\psi} v+V\left(p_{x}, p_{y}\right)\left(\begin{array}{c}
\cos \psi \\
\sin \psi
\end{array}\right)-1,
$$

where equations for adjoint variables $p_{x}, p_{y}$ and $p_{\psi}$ are given by

$$
\begin{aligned}
& \dot{p}_{x}=0, \quad \dot{p}_{y}=0, \\
& \dot{p}_{\psi}=V\left(p_{x}, p_{y}\right)\left(\begin{array}{c}
\sin \psi \\
-\cos \psi
\end{array}\right) .
\end{aligned}
$$

The optimal control for the time-minimum problem now is given by

$$
v=\Xi \operatorname{sign}\left(p_{\psi}\right) .
$$

By differentiating the expression for $\dot{p}_{\psi}$ (3) and combining it with Hamiltonian (2) for both cases when $p_{\psi}>0$ and $p_{\psi}<0$ we can get a set of equations for $p_{\psi}:$

$$
\ddot{p}_{\psi}+\Xi^{2} p_{\psi} \mp \Xi=0 \text {. }
$$

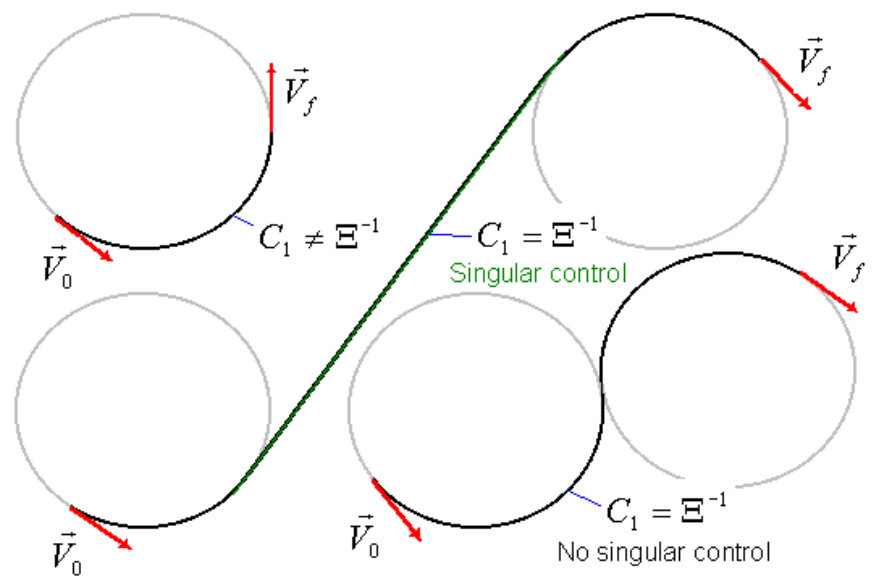

Figure 4 Planar projections of possible trajectories
This differential equation gives two sinusoids (shifted with respect to abscise axis by $\pm \Xi^{-1}$ ) as solutions for the general (non-singular) case

$$
p_{\psi}=C_{1} \sin \left(\Xi t+C_{2}\right) \pm \Xi^{-1},
$$

where $C_{1}$ and $C_{2}$ are constants defined by the concrete boundary conditions. If $C_{1} \neq \Xi^{-1}$ the parafoil model moves along a descending spiral. It takes $2 \pi \Xi^{-1}$ seconds to make a full turn with a radius of $V \Xi^{-1}$. If $C_{1}=\Xi^{-1}$ there exists a possibility of singular control. This is caused by the fact that there exists a point in time where both $p_{\psi}$ and $\dot{p}_{\psi}$ are zero as can be seen from (6).

Consider singular control for this model. By definition it means that $p_{\psi} \equiv 0$. For the time-optimal problem from the Hamiltonian (2) and third equation in (3) (of course keeping in mind the first two) it follows that for a singular control case

$$
p_{x}=V^{-1} \cos \psi, p_{y}=V^{-1} \sin \psi, \psi=\text { const } .
$$

Expressions (7) imply that singular control corresponds to motion with a constant heading $(v \equiv 0)$. It may not however be realized. Instead, the parafoil model may switch from right-handed spiral to a lefthanded one or vice versa. Planar projections of possible trajectories are shown on Fig.4. Concrete boundary conditions define which case will be realized.
To summarize, Figs.5,6 show examples of threedimensional (3D) time-optimal trajectories computed in the inertial frame for the gliding system (1). As suggested by the Pontrjagin's principle of optimality they would consist of helices (spiral descent) and straight descent segments. The basic difference between two trajectories shown on Fig.5 is whether parafoil expends its potential energy at the beginning of descent or at the end. It may depend on the tactical conditions in the LZ, terrain, or some other factors. In a general case parafoil could spend its potential energy in the vicinity of some other WP differing from the start and final portions of the trajectory as it shown in Fig.6. In any case, since the control system cannot meet any time constraints due to 
unavailability of thrust on a parafoil system, the common feature of these RTs is that they are defined in the inertial frame and are time independent.

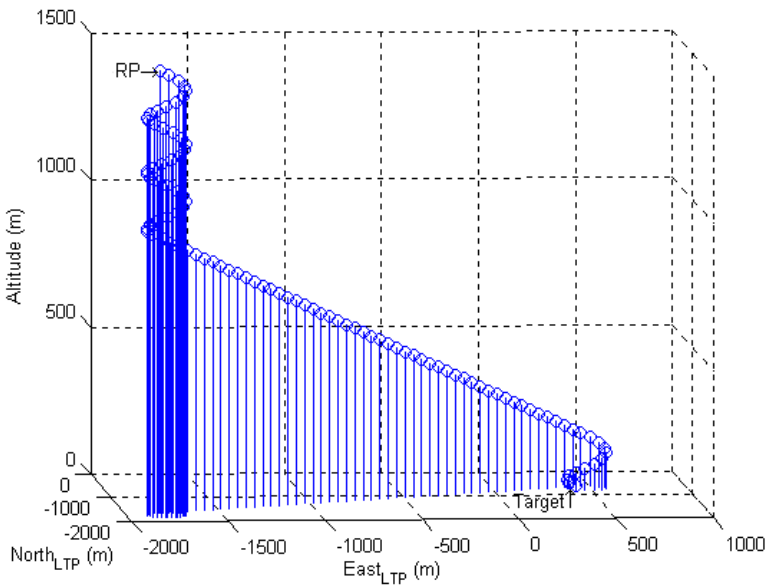

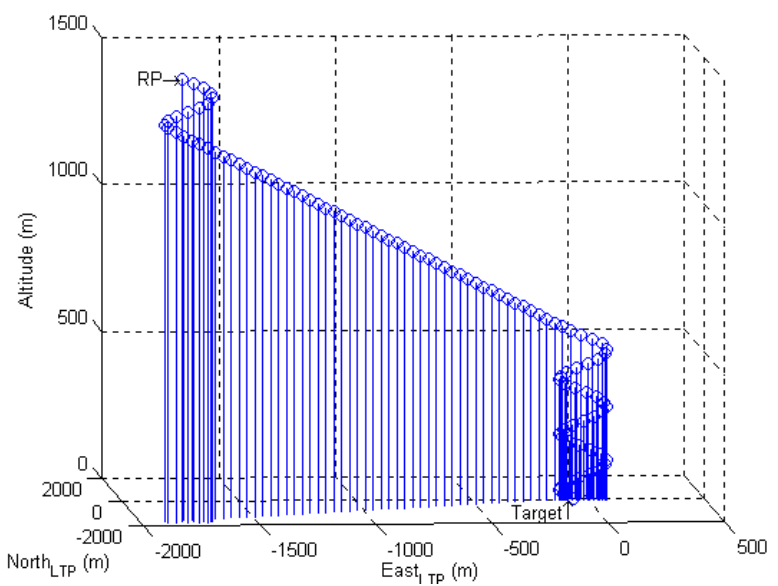

Figure $53 \mathrm{D}$ representation of possible inertial RTs

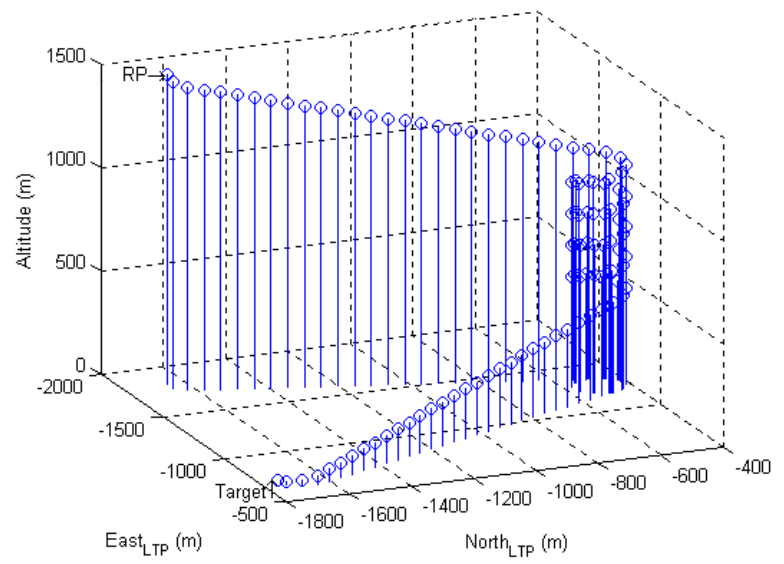

Figure 6 General-case trajectory

Another critical issue is that in order to meet a softlanding requirement the parafoil at the LZ must align itself into the wind.

\section{Real-Time Trajectory Generation}

The optimal control analysis of the previous section motivated the basic RT structure as is shown in Fig. 7. This RT consists of three segments: initial straight-line glide (segment 1), spiral descent (segment 2) and final glide and flare (segment 3 ). Since it is required that the parafoil is aligned into the wind at LZ, the final glide segment ends at the desired impact point (DIP) and is directed into the wind. Furthermore, the final glide starts an offset distance $d_{\text {offset }}$ away and a certain height above DIP. The height is determined by the flight path angle of the segment 3. Similarly, the first segment starts at the parafoil release point (RP) and ends at a point defined by the flight path angle of the first segment at the spiral descent segment. The first and last segments are fused together by the spiral descent seg- ment. The radius of the spiral is adjusted to provide smooth transition between each segment. The rest of the section derives the mathematical representation of the complete RT.

Let $\gamma_{R T_{1}}$ denote the desired flight path angle for the first straight-line segment of the trajectory, similarly let $\gamma_{R T_{2}}$ denote the flight path angle for the spiral-descent segment and, finally, let $\gamma_{R T_{3}}$ represent the flight path angle for the final straight-line segment. Furthermore, let $\psi_{f}$ represent the wind direction at the LZ, $\mathbf{p}_{0}=\left(x_{0}, y_{0}, z_{0}\right)^{T}$ is the RP, and $\mathbf{p}_{f}=\left(x_{f}, y_{f}, z_{f}\right)^{T}$ denotes the DIP. Define vector $\mathbf{i}=\left(\cos \left(\psi_{f}+\pi\right), \sin \left(\psi_{f}+\pi\right), 0\right)^{T}$ to be the unit vector that points into the wind at the LZ and vector $\mathbf{i}^{*}=\left(-\sin \left(\psi_{f}+\pi\right), \cos \left(\psi_{f}+\pi\right), 0\right)^{T}$ to be the unit vec- 
tor orthogonal to i. Then the initial point $\mathbf{p}_{3}=\left(x_{3}, y_{3}, z_{3}\right)^{T}$ of the third segment is given by

$$
\mathbf{p}_{3}=-d_{\text {offset }} \mathbf{i}+\left(0,0, z_{f}+d_{\text {offset }} \tan \gamma_{R T_{3}}\right)^{T} .
$$
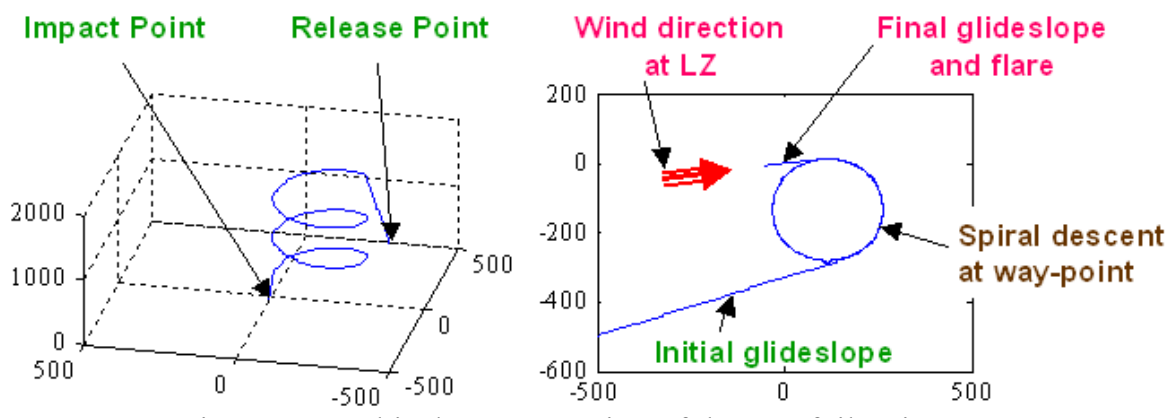

Figure 7 Graphical representation of the parafoil trajectory

Now, let $\mathbf{p}_{R T}$ denote the position on the RT, $s$ denote the path length traveled along the RT and $V_{R T}$ parafoil's velocity along this path. Then the expression for the third segment of the desired trajectory is

$$
\mathbf{p}_{R T}(s)=\mathbf{p}_{3}+\mathbf{T}_{3} s, \dot{s}=V_{R T},
$$

where

$$
\mathbf{T}_{3}=\left(-\cos \psi_{f} \cos \gamma_{R T_{3}},-\sin \psi_{f} \cos \gamma_{R T_{3}},-\sin \gamma_{R T_{3}}\right)^{T}
$$

is the unit vector that points along the line connecting $\mathbf{p}_{3}$ to $\mathbf{p}_{f}$. Note, that the length of the third segment $s_{3}=\left\|\mathbf{p}_{3}-\mathbf{p}_{f}\right\|$. Similarly, let $s_{1}$ denote the length of the first segment and $s_{2}$ - of the second. Then, along the third segment $\left(s_{1}+s_{2}<s \leq s_{1}+s_{2}+s_{3}\right)$

$$
\mathbf{p}_{R T}(s)=\mathbf{p}_{3}+\mathbf{T}_{3}\left(s-s_{1}+s_{2}\right) \text {. }
$$

Next, consider Fig.8. Let $r$ represent the radius of the spiral descent segment. Then the center of the spiral $\mathbf{p}_{1}=\left(x_{1}, y_{1}, z_{1}\right)^{T}$ has the following expression

$$
\mathbf{p}_{1}=\mathbf{p}_{3}+r \mathbf{i}^{*},
$$

which is used next to determine the expression for $\mathbf{p}_{R T}$ along segment 1 . Let $d=\sqrt{\left(x_{0}-x_{1}\right)^{2}+\left(y_{0}-y_{1}\right)^{2}}$, then $\Delta \psi=\sin ^{-1} \frac{r}{d}, \psi_{1}=\tan ^{-1} \frac{y_{1}-y_{0}}{x_{1}-x_{0}}$ and $\psi_{2}=\psi_{1}+\Delta \psi$.

Analogous to (10) define

$$
\mathbf{T}_{1}=\left(\cos \psi_{2} \cos \gamma_{R T_{1}}, \sin \psi_{2} \cos \gamma_{R T_{1}},-\sin \gamma_{R T_{1}}\right)^{T} \text {. }
$$

Then

$$
\mathbf{p}_{2}=\mathbf{p}_{0}+\mathbf{T}_{1} \frac{\sqrt{d^{2}-r^{2}}}{\cos \gamma_{R T_{1}}}, s_{1}=\left\|\mathbf{p}_{0}-\mathbf{p}_{2}\right\|
$$

and

$$
\mathbf{p}_{R T}(s)=\mathbf{p}_{0}+\mathbf{T}_{1} s, 0 \leq s \leq s_{1} .
$$

The turn rate $\dot{\psi}_{R T}$ along the spiral descent segment is given by

$$
\dot{\psi}_{R T}=V_{R T} r^{-1} \cos \gamma_{R T_{2}}
$$

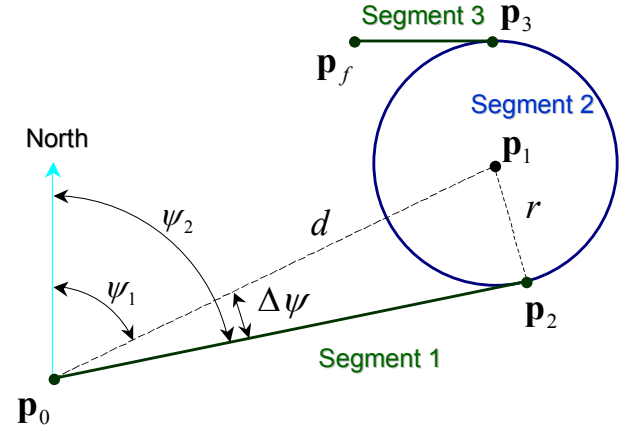

Figure 8 Top view of the parafoil RT

Then, along the segment $2\left(s_{1}<s \leq s_{1}+s_{2}\right)$

$$
\mathbf{p}_{R T}(s)=\mathbf{p}_{1}+\left(\begin{array}{c}
r \sin \left(\rho_{R T}^{*}\left(s-s_{1}\right)+\Delta\right) \\
-r \cos \left(\rho_{R T}^{*}\left(s-s_{1}\right)+\Delta\right) \\
\left(s_{1}-s\right) \sin \gamma_{R T_{2}}
\end{array}\right)
$$

where $\quad \rho_{R T}^{*}=\dot{\psi}_{R T} V_{R T}^{-1}, \quad \Delta=\psi_{f}+\pi-\rho_{R T}^{*}\left(s_{2}-s_{1}\right)$, $s_{2}=\frac{z_{2}-z_{3}}{\sin \gamma_{R T_{2}}}$

Without loss of generality, the $z$-component of the center of the spiral descent $\mathbf{p}_{1}$ was set to equal $z_{2}$ in the definition of $\mathbf{p}_{R T}(s)$ above.

$$
\mathbf{T}_{2}(s)=\frac{\frac{d \mathbf{p}_{R T}}{d s}}{\left\|\frac{d \mathbf{p}_{R T}}{d s}\right\|}=\left(\begin{array}{c}
\cos \left(\rho_{R T}^{*}\left(s-s_{1}\right)+\Delta\right) \cos \gamma_{R T_{2}} \\
\sin \left(\rho_{R T}^{*}\left(s-s_{1}\right)+\Delta\right) \cos \gamma_{R T_{2}} \\
-\sin \gamma_{R T_{2}}
\end{array}\right)
$$

Then, the choice of $\Delta$ guarantees that the horizontal projections of vectors defined by Eqns. (18) and (10) are aligned, i.e.

$$
\left.\mathbf{T}_{2}^{h o r}\right|_{s=s_{2}}=\left.\mathbf{T}_{3}^{h o r}\right|_{s=s_{2}}=\left(-\cos \psi_{f},-\sin \psi_{f}\right)^{T} .
$$


This fact implies that at $\mathbf{p}_{3}$ the horizontal projections of the commanded velocity vectors for segments 2 and 3 are equal and are independent of the choice of $r$.

Finally, the radius of the spiral descent $r$ is selected to guarantee that at $\mathbf{p}_{2}$ segments 1 and 2 merge smoothly. This is done numerically by solving a single variable constrained optimization problem. Note that at $\mathbf{p}_{2}$

$$
\left.\mathbf{p}_{R T}\right|_{s=s_{1}}=\mathbf{p}_{2}=\mathbf{p}_{1}+(r \sin \Delta,-r \cos \Delta, 0)^{T} .
$$

Therefore, let

$$
e(r) \doteq\left\|\mathbf{p}_{2}-\mathbf{p}_{1}+(r \sin \Delta,-r \cos \Delta, 0)^{T}\right\|,
$$

then the desired value of $r$ is $r_{d e s}=\underset{r_{\min } \leq r \leq r_{\max }}{\arg \min } e(r)$. Fig.9 includes a plot of $e(r)$ versus $r$, which indicates that there are multiple values of $r_{\text {des }}$. Therefore the values of $r_{\min }$ and $r_{\max }$ are selected to provide a unique solution that is consistent with physical limitations of the parafoil. Note, in steady state turn the bank angle of the parafoil is defined by the following expression:

$$
\phi_{R T}=\tan ^{-1} \frac{V_{R T} \dot{\psi}_{R T}}{g}=\tan ^{-1}\left(\frac{V_{R T}^{2} \cos \gamma_{R T_{2}}}{r g}\right),
$$

where $g$ is acceleration due to gravity. By construction the horizontal projections of $\mathbf{T}_{1}$ and $\mathbf{T}_{2}$ at $\mathbf{p}_{2}$ are aligned, i.e. $\left.\mathbf{T}_{1}^{h o r}\right|_{s=s_{1}}=\left.\mathbf{T}_{2}^{h o r}\right|_{s=s_{1}}$.

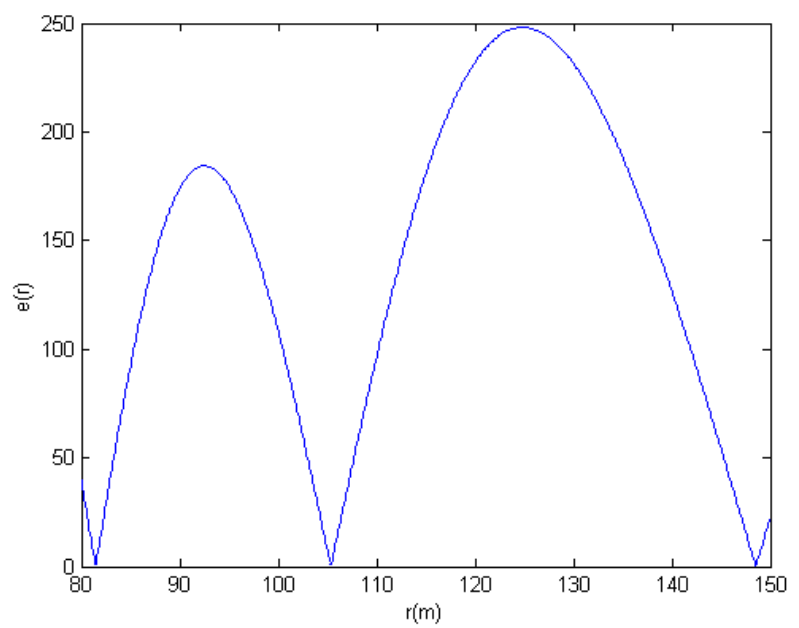

Figure 9 A representative plot of $e(r)$ versus $r$

\section{Integrated Guidance and Control Algorithm}

The development of the integrated guidance and control algorithm is based on the work reported in Ref.34, where authors have proposed a new technique for tracking so-called trimming trajectories for UAV's. As shown in Ref.34 trimming trajectories consist of straight lines and helices. Therefore, this methodology is particularly suitable for the problem at hand.

The key ideas of the design methodology developed in Ref.34 include the following five steps:

1) reparameterize trimming trajectory using the arclength $s$, thus eliminating time as an independent variable;

2) resolve the position and velocity errors in so-called Frenet frame (Ref.34);

3) form error dynamics for the system consisting of the trajectory and parafoil model, where the position and velocity error states are resolved in Frenet frame;

4) design a linear tracking controller for the linearization of the system along the trimming trajectory;

5) implement this controller with the true nonlinear plant using a nonlinear transformation provided in Ref.34. This implementation guarantees that the linearization along the trimming trajectory of the feedback system consisting of the nonlinear plant and nonlinear controller preserves the eigenvalues and transfer functions of the feedback interconnection of the linearized error dynamics and linear tracking controller (see Ref.36).

\section{V.1 Derivation of the errors in Frenet frame}

As shown in Section IV the desired RT is parameterized using the path/arclength parameter $s$.

Let $\mathbf{p}=(x, y, z)^{T}$ denote current position of the parafoil. The methodology developed in Ref.34 requires that position and velocity commands $\mathbf{p}_{R T}\left(s_{*}\right), V_{R T}\left(s_{*}\right)$ used to compute position and velocity errors, $\mathbf{p}_{R T}\left(s_{*}\right)-\mathbf{p}$ and $V_{R T}\left(s_{*}\right)-V$ respectively, correspond to the point on the RT that is nearest to current position of the vehicle. This is done by first determining the value of the path/arclength parameter $S_{*}=\arg \min \left\|\mathbf{p}_{R T}(s)-\mathbf{p}\right\|^{2}$ and then using it to compute position and velocity commands. In Ref.34 the problem of determining $s_{*}$ is reduced to a constrained optimization problem. However, computing limitations of the onboard processor have imposed a need to develop analytical techniques for the computation of $s_{*}$, discussed next.

An exact analytical expression for $s_{*}$ can be derived for straight-line segments 1 and 3. Recall Eqns. (15) and (11). Then 


$$
s_{*}=\underset{s}{\arg \min }\left(\begin{array}{ll}
\left\|\mathbf{p}_{0}+\mathbf{T}_{1} s-\mathbf{p}\right\|, & 0 \leq s \leq s_{1} \\
\left\|\mathbf{p}_{3}+\mathbf{T}_{3}\left(s-s_{1}-s_{2}\right)-\mathbf{p}\right\|, & s_{1}+s_{2}<s \leq s_{1}+s_{2}+s_{3}
\end{array}\right)
$$
ments

Simple algebra shows that for straight-line seg-

$$
s_{*}=\left\{\begin{array}{l}
\mathrm{T}_{1}^{T}\left(\mathrm{p}-\mathrm{p}_{0}\right), 0 \leq s \leq s_{1}, \\
\mathrm{~T}_{3}^{T}\left(\mathrm{p}-\mathrm{p}_{3}\right)+\left(s_{1}+s_{2}\right), s_{1}+s_{2}<s \leq s_{1}+s_{2}+s_{3} .
\end{array}\right.
$$

Now, recall that segment 2 represents a spiral. In this case, only an approximation of $S_{*}$ can be found analytically. Its derivation is discussed next.

Let

$$
C=\left\{(l, m, n):\left(l-x_{1}\right)^{2}+\left(m-y_{1}\right)^{2}=r^{2}, z_{1} \leq n \leq z_{2}\right\}
$$

define a cylinder centered at $\mathbf{p}_{1}=\left(x_{1}, y_{1}, z_{1}\right)^{T}$. Then the spiral descent trajectory

$$
\mathbf{p}_{R T}(s)=\mathbf{p}_{1}+\left(\begin{array}{c}
r \sin \left(\rho_{R T}^{*}\left(s-s_{1}\right)+\Delta\right) \\
-r \cos \left(\rho_{R T}^{*}\left(s-s_{1}\right)+\Delta\right) \\
-\left(s-s_{1}\right) \sin \gamma_{R T_{2}}
\end{array}\right)
$$

$\left(s_{1}<s \leq s_{1}+s_{2}\right)$ is a subset of $C$. Furthermore, the projection of the vehicle's position $\mathbf{p}$ onto $C$ is

$$
\mathbf{p}_{\text {proj }}=\left(\left(x_{1}, y_{1}\right)+r \frac{\left(x-x_{1}, y-y_{1}\right)}{\left\|x-x_{1}, y-y_{1}\right\|}, z\right)^{T}
$$

For any vector $\mathbf{p}_{c y l}=\left(x_{c y l}, y_{c y l}, z_{c y l}\right)^{T} \in C$ define a function $\Pi: R^{3} \rightarrow R^{2}$,

$$
\Pi\left(\mathbf{p}_{c y l}\right)=:\left(\begin{array}{c}
x_{\Pi} \\
z_{\Pi}
\end{array}\right)=\left(\begin{array}{r}
r \tan ^{-1}\left(\frac{x_{1}-x_{c y l}}{y_{1}-y_{c y l}}+\pi\right) \\
z_{c y l}
\end{array}\right) .
$$

The function $\Pi$ maps the cylinder $C$ onto a rectangle of width $2 \pi r$ and height $z_{2}-z_{1}$. Moreover, the trajectory $\mathbf{p}_{R T}(s)$ defined for segment 2 is mapped into a function $\Pi\left(\mathbf{p}_{R T}(s)\right)$ shown in Fig. 10 .

Let $d_{*}$ denote the distance from $\mathbf{p}$ to the nearest point $\mathbf{p}_{R T}\left(s_{*}\right)$ on the trajectory $\mathbf{p}_{R T}(s)$. Then $\mathbf{p}_{R T}\left(s_{*}\right)$ can be obtained by determining the intersection of the ball $B_{d_{*}}$ of radius $d_{*}$ centered at $\mathbf{p}$. The mapping $\Pi\left(B_{d_{*}} \cap C\right)$ is an ellipse centered at $\Pi\left(\mathbf{p}_{\text {proj }}\right)$ with semi-major and semi-minor radii given by $r_{z}=d_{x} \sqrt{3}$ and $r_{x}=r \cos ^{-1} \frac{r^{2}+\left(r+d_{x}\right)^{2}-4 d_{x}^{2}}{2 r\left(r+d_{x}\right)}$, respectively. In the previous expressions $d_{x}=\left(x-x_{\text {proj }}\right)^{2}+\left(y-y_{\text {proj }}\right)^{2}$. Intersection of this ellipsoid with $\Pi(\mathbf{p}(s))$ represents the mapping $\Pi\left(\mathbf{p}\left(s_{*}\right)\right)$ of $\mathbf{p}\left(s_{*}\right)$. Analytical approximation of $\Pi\left(\mathbf{p}\left(s_{*}\right)\right)$ is obtained next and then used to compute $S_{*}$.

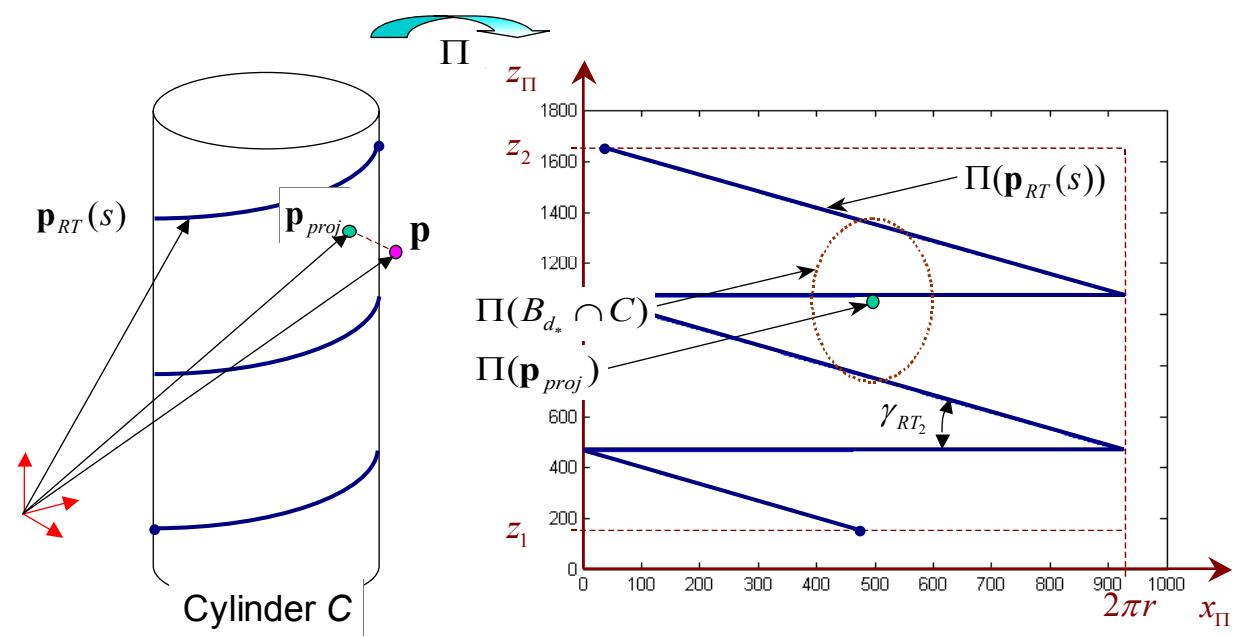

Figure 10 Graphical representation of $\Pi(C)$

Consider Fig.11. Note for the case illustrated in this figure, the $z_{\Pi}$ coordinate of $\Pi\left(\mathbf{p}\left(s_{*}\right)\right)$ is bounded above by $z_{1 n}$ and below by $z_{1 p}$. Using the basic geometry shown in Fig.11, expressions for $z_{1 n}$ and $z_{1 p}$ are:

$$
\begin{aligned}
& z_{1 n}=z_{\text {proj }}+\left|\bmod \left(z_{0 n}-z_{\text {proj }}, 2 \pi r \tan \gamma_{R T_{2}}\right)\right|, \\
& z_{1 p}=z_{\text {proj }}+\left|z_{1 n}-z_{p r o j}\right| \cos ^{2} \gamma_{R T_{2}},
\end{aligned}
$$




$$
\begin{aligned}
& z_{0 \mathrm{n}}=z_{1}-s_{* n} \sin \gamma_{\mathrm{RT}_{2}}, \\
& s_{* n}=\rho_{R T}^{*}\left(\frac{x_{p r o j}}{r}-\bmod (\Delta, 2 \pi)\right) .
\end{aligned}
$$

Similarly,

$$
\begin{aligned}
& z_{2 n}=z_{1 n}-2 \pi r \tan \gamma_{R T_{2}}, \\
& z_{2 p}=z_{p r o j}-\left|z_{2 n}-z_{p r o j}\right| \cos ^{2} \gamma_{R T_{2}} .
\end{aligned}
$$

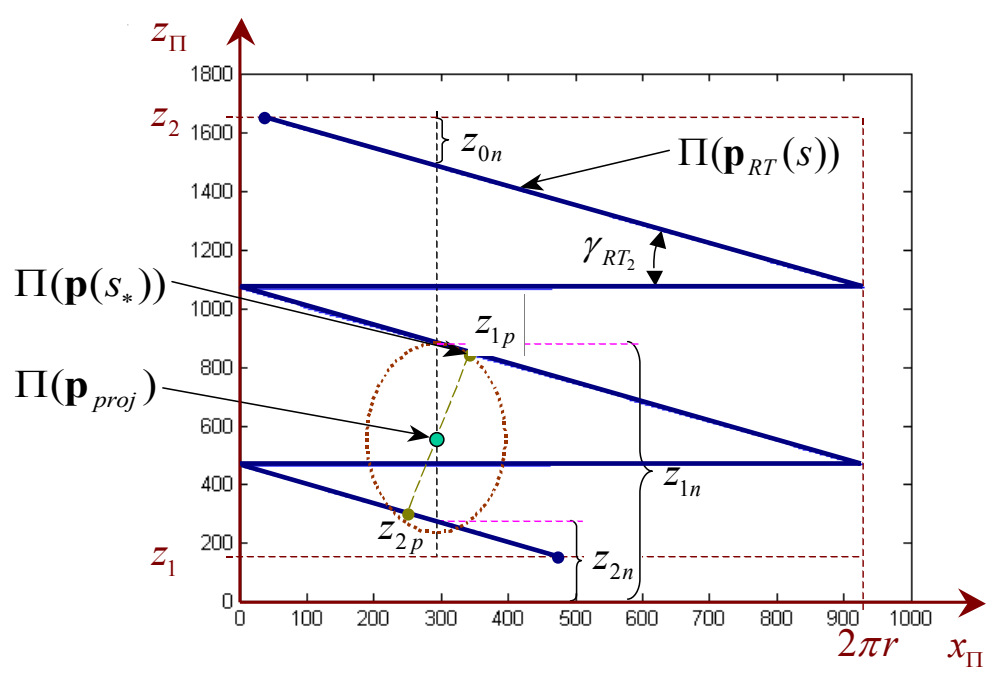

Figure 11 Graphical representation of the range of the function $\Pi$

The variables $z_{2 p}$ and $z_{1 p}$ represent the $z$ coordinates of the projections of $\Pi\left(\mathbf{p}_{\text {proj }}\right)$ onto the two nearest legs of $\Pi(\mathbf{p}(s))$. These variables can now be used to find the approximation of $z_{\Pi}, z_{a p p}$ :

$$
z_{a p p}= \begin{cases}z_{1 p}, & \text { if }\left|z_{1 p}-z_{p r o j}\right|<\left|z_{2 p}-z_{p r o j}\right| \\ z_{a p p}=z_{2 p}, & \text { otherwise. }\end{cases}
$$

Note that logic (32) guarantees that in the case when two legs of $\mathbf{p}(s)$ are equidistant from $\mathbf{p}$, the point closer to the ground is selected. Furthermore, since $\Pi$ maps $z$-axis of $R^{3}$ onto itself $z_{a p p}$ can be used to compute $S_{*}$

$$
S_{*}=\frac{z_{1}-z_{a p p}}{\sin \gamma_{R T_{2}}},
$$

which follows from the definition of $\mathbf{p}(s)$ for the spiral descent segment. This value of $s_{*}$ can now be used to compute the unit basis vectors $\mathbf{T}_{2}, \mathbf{N}_{2}$, and $\mathbf{B}_{2}$ of the Frenet frame $\{F\}$ for segment 2 at $s=s_{*}$

$$
\mathbf{T}_{2}=\frac{\frac{d \mathbf{p}_{c}}{d s}}{\left\|\frac{d \mathbf{p}_{c}}{d s}\right\|}, \quad \mathbf{N}_{2}=\frac{\frac{d \mathbf{T}_{2}}{d s}}{\left\|\frac{d \mathbf{T}_{2}}{d s}\right\|}, \quad \mathbf{B}_{2}=\frac{\mathbf{T}_{2} \times \mathbf{N}_{2}}{\left\|\mathbf{T}_{2} \times \mathbf{N}_{2}\right\|}
$$

or in the final form

$$
\begin{aligned}
& \mathbf{T}_{2}\left(s_{*}\right)==\left(\begin{array}{c}
\cos \left(\rho_{R T}^{*}\left(s_{*}-s_{1}\right)+\Delta\right) \cos \gamma_{R T_{2}} \\
\sin \left(\rho_{R T}^{*}\left(s_{*}-s_{1}\right)+\Delta\right) \cos \gamma_{R T_{2}} \\
-\sin \gamma_{R T_{2}}
\end{array}\right), \\
& \mathbf{N}_{2}\left(s_{*}\right)==\left(\begin{array}{c}
-\sin \left(\rho_{R T}^{*}\left(s_{*}-s_{\lim _{1}}\right)+\Delta\right) \\
\cos \left(\rho_{R T}^{*}\left(s_{*}-s_{1}\right)+\Delta\right) \\
0
\end{array}\right), \\
& \mathbf{B}_{2}\left(s_{*}\right)=\frac{\mathbf{T}_{2}\left(s_{*}\right) \times \mathbf{N}_{2}\left(s_{*}\right)}{\left\|\mathbf{T}_{2}\left(s_{*}\right) \times \mathbf{N}_{2}\left(s_{*}\right)\right\|} .
\end{aligned}
$$

For segments 1 and 3 the basis vectors are constant. For example, for segment 1 we obtain

$$
\mathbf{T}_{1}=\left(\begin{array}{c}
\cos \psi_{2} \cos \gamma_{R T_{1}} \\
\sin \psi_{2} \cos \gamma_{R T_{1}} \\
-\sin \gamma_{R T_{1}}
\end{array}\right), \mathbf{N}_{1}=\left(\begin{array}{c}
-\sin \psi_{2} \\
\cos \psi_{2} \\
0
\end{array}\right) .
$$

And for segment 3,

$$
\mathbf{T}_{3}=\left(\begin{array}{c}
-\cos \psi_{f} \cos \gamma_{R T_{3}} \\
-\sin \psi_{f} \cos \gamma_{R T_{3}} \\
-\sin \gamma_{R T_{3}}
\end{array}\right), \mathbf{N}_{3}=\left(\begin{array}{c}
\sin \psi_{f} \\
-\cos \psi_{f} \\
0
\end{array}\right) .
$$

The basis vectors $\mathbf{B}_{1}$ and $\mathbf{B}_{3}$ are computed exactly as $\mathbf{B}_{2}$. Now the rotation matrix $\underset{L T P}{\stackrel{F}{R} R_{i}}$ from LTP to Frenet frame for the $i$-th segment is given by ${ }_{L T P}^{F} R_{i}=\left(\mathbf{T}_{i}, \mathbf{N}_{i}, \mathbf{B}_{i}\right)^{T}$ and the corresponding position and velocity errors are 


$$
\begin{aligned}
& \mathbf{p}_{e}=\underset{L T P}{{ }_{L T P}^{F}} R_{i}\left(\mathbf{p}_{R T}\left(s_{*}\right)-\mathbf{p}\right)=:\left(0, y_{e}, z_{e}\right)^{T}, \\
& \dot{\mathbf{p}}_{e}={ }_{L T P}^{F} R_{i}\left(\dot{\mathbf{p}}_{R T}\left(s_{*}\right)-\mathbf{V}\right)+{ }_{L T P}^{F} \dot{R}_{i}\left(\mathbf{p}_{R T}\left(s_{*}\right)-\mathbf{p}\right) .
\end{aligned}
$$

Notice that by construction the $x$-component of the position error vector $\mathbf{p}_{e}$ is zero, see Ref.34. These error vectors were used to design a tracking control system discussed in the next section.

\section{V.2 Control System Design}

The trajectory tracking controller design methodology proposed in Ref.34 is now applied to the design of a tracking controller for the trajectory generated using the algorithm developed in Section IV. The controller can only use GPS position and velocity for feedback. This constraint is motivated by the requirement to keep the cost of the onboard avionics low, therefore, only GPS is available for control.

During segments 1 and 2 the control system uses differential flaps to drive the lateral components of the position and velocity error vectors to zero. On the other hand, the last segment is tracked using both symmetric and differential flaps, denoted here by $\delta_{S}$ and $\delta_{D}$, respectively. The structure of tracking controller for the lateral channel shown is Fig. 12.

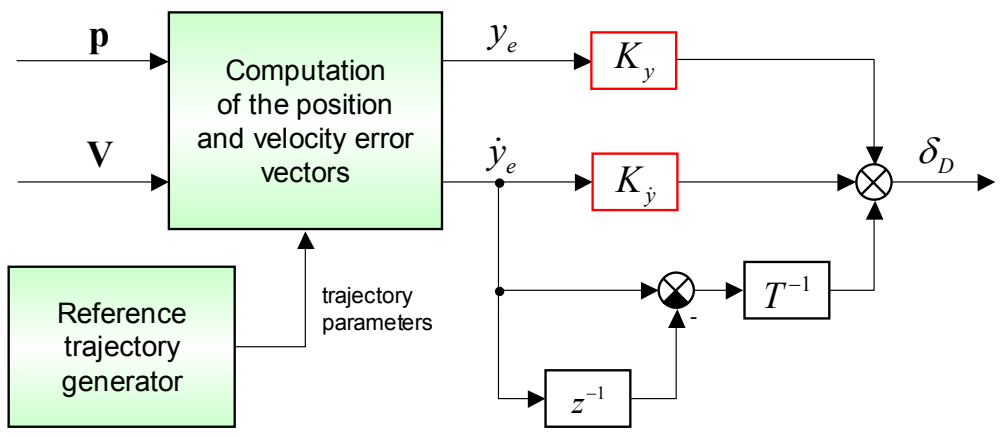

Figure 12 Lateral channel tracking controller structure

The gains $K_{y}$ and $K_{\dot{y}}$ shown in Fig.12 were selected to provide stability and performance for the lateral channel of the feedback system. The variables $z^{-1}$ and $T$ denote backward shift and sampling period. The sampling period for this problem was dictated by the onboard GPS receiver, whose update rate is $2 \mathrm{~Hz}$.

The structure of the vertical channel controller is shown in Fig.13. This controller uses symmetric flaps $\delta_{S}$ to drive the vertical channel error to 0 and was only used to track the final segment of the trajectory. This decision was motivated by the fact that symmetric flaps have negligible control authority in spiral descent. Furthermore, deflecting symmetric flaps tends to increase the descent rate of the parafoil while reducing the flap deflection budget available for differential flaps command and, therefore, symmetric flaps were not used during segment 1 as well.

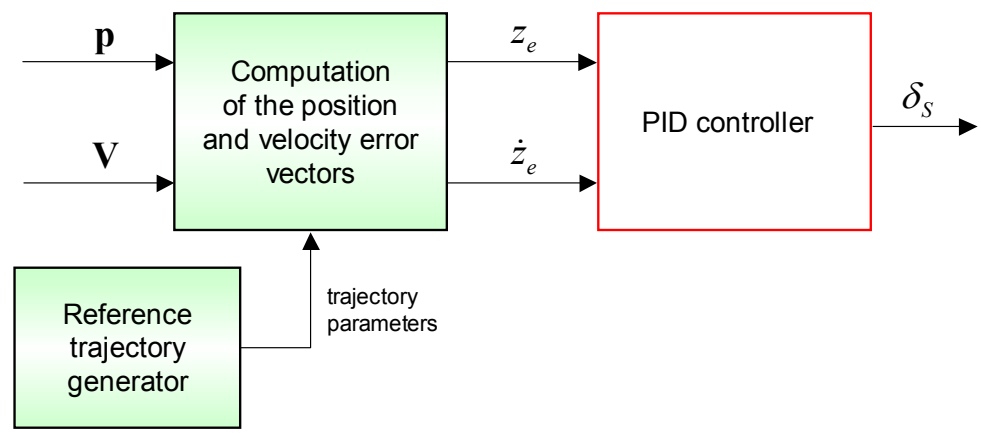

Figure 13 Vertical channel tracking controller structure

\section{Simulation Results}

The trajectory tracking control system discussed in Section $\mathrm{V}$ has been tested in simulation using a nonlinear 6DOF model of the Pegasus parafoil that also includes the available actuator model. ${ }^{35}$ Fig. 14 shows a compari- son between flight test data collected during Pegasus drop at the YPG and model's response to the wind collected during the same drop. Clearly, the 6DOF model provides a reasonable approximation of the parafoil dynamics. 


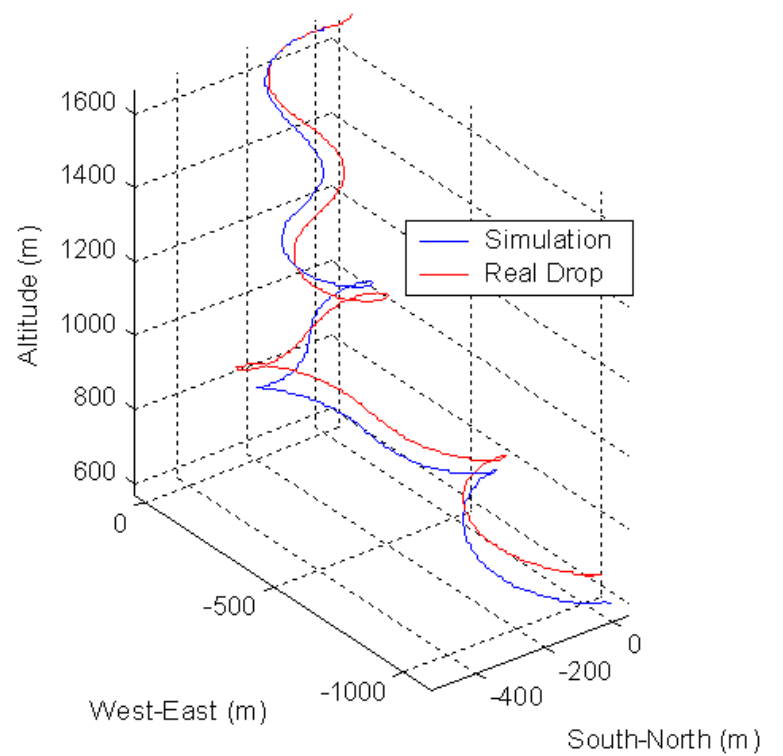

Figure 14 Comparison of the flight test data and simulation results
Fig. 15 shows results of an extensive simulation analysis of the trajectory tracking control system using the 6DOF model discussed above. Each of 40 simulation runs included the wind profile obtained at YPG. The GPS position and velocity errors were modeled as white noise processes with zero mean and standard deviations shown in Tab.1. Finally, landing performance statistics shown in Tab.2 indicate that system's performance exceeded the required circular error probable (CEP) of $100 m$.

Table 1 GPS errors (STD)

\begin{tabular}{lccc}
\hline \hline & $x$-direction & $y$-direction & $z$-direction \\
\hline Position $(m)$ & 5 & 5 & 10 \\
Velocity $(m / s)$ & 0.3 & 0.3 & 0.5 \\
\hline \hline
\end{tabular}

Table 2 Touchdown errors

\begin{tabular}{ccc}
\hline \hline CEP $(m)$ & Mean value $(m)$ & STD $(m)$ \\
\hline 70.7 & 67.5 & 20.5 \\
\hline \hline
\end{tabular}

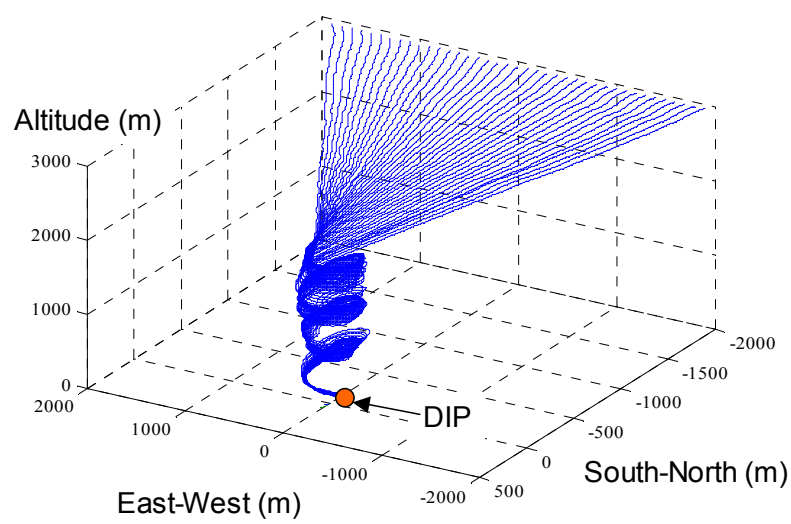

Figure 15 Simulation results of the trajectory tracking control system

\section{Conclusions}

The optimal control strategy for the Pegasus parafoil payload delivery system was synthesized based on Pontrjagin's maximum principle. This motivated the structure of the real-time reference trajectory generator. Together with the robust path following algorithm it enabled a successful development and simulation testing of the complete guidance and control algorithm. The authors plan to flight test developed algorithms in the early spring of 2003 and to have complete flight test results soon afterwards.

\section{References:}

1. Forehand, J.E., "The Precision Drop Glider (PDG)," Proceedings of the Symposium on Parachute Technology and Evaluation, El Centro, CA, April 1969 (USAF report FTC-TR-64-12, pp.24-44).
2. Poyner, D. The Parachute Manual, Para Publishing, Santa Barbara, CA, Vol II, $4^{\text {th }}$ edition, 1991.

3. Knacke, T.W. Parachute Recovery Systems Design Manual. Para Publishing, Santa Barbara, CA, 1992.

4. Lavitt, M.O., "Fly-by-Wire Parafoil Can Deliver 1,100 lbs," Aviation Week and Space Technology, Vol.144 (14), 1996, pp.64-64.

5. Wytlie, T., and Downs, P., "Precision Parafoil Recovery - Providing Flexibility for Battlefield UAV Systems?," Proceedings of the $14^{\text {th }}$ AIAA Aerodynamic Decelerator Systems Technology Conference, San Francisco, CA, June 3-5, 1997.

6. Wyllie, T., "Parachute Recovery for UAV Systems," Aircraft Engineering and Aerospace Technology, vol.73 (6), 2001, pp.542-551.

7. Wailes, W., and Hairington, N., "The Guided Parafoil Airborne Delivery System Program", Proceedings of the $13^{\text {th }}$ AIAA Aerodynamic Decelerator Systems Technology Conference, Clearwater Beach, FL, May 15-18, 1995. 
8. Patel, S., Hackett, N.R., and Jorgensen D.S., "Qualification of the Guided parafoil Air Delivery System-Light (GPADS-Light)," Proceedings of the $14^{\text {th }}$ AIAA Aerodynamic Decelerator Systems Technology Conference, San Francisco, CA, June 3-5, 1997.

9. Smith, J., Bennett, B., and Fox R., "Development of the NASA X-38 Parafoil Landing System," Proceedings of the $3^{\text {rd }}$ AIAA Workshop on Weakly Ionized Gases Workshop, Norfolk, VA, November 1-5, 1999.

10. Smith, B.A., "Large X-38 Parafoil Passes First Flight Test," Aviation Week and Space Technology, vol.152 (5), 2000, pp.40-44.

11. Machin, R.A., Iacomini, C.S., Cerimele, C.J., et al., "Flight Testing the Parachute System for the Space Station Crew Return Vehicle," Journal of Aircraft, vol.38 (5), 2001, pp.786-799.

12. Smith, J., Witkowski, A., and Woodruff P., "Parafoil Recovery Subsystem for the Genesis Space Return Capsule," Proceedings of the $16^{\text {th }}$ AIAA Aerodynamic Decelerator Systems Technology Conference, Boston, MA, May 21-24, 2001.

13. Smith, B.D., "Steering Algorithms for GPS Guidance of RAM-AIR Parachutes," Proceedings the $8^{\text {th }}$ International Technical Meeting of the Institute of Navigation (IONGPS-95), Palm Springs, CA, September 12-15, 1995.

14. Murray, J.E., Sim, A.G., et al, "Further Development and Flight Test of an Autonomous Precision Landing System Using a Parafoil," NASA Technical Memorandum \#4599, 1994.

15. Sim, A.G., Murray J.E., Neufeld, D.C., et al., "Development and Flight Test of a Deployable Precision Landing System," Journal of Aircraft, vol.31 (5), 1994, pp.11011108.

16. Hogue, J.R., and Jex, H.R., "Applying Parachute Canopy Control and Guidance Methodology to Advanced Precision Airborne Delivery Systems," Proceedings of the $13^{\text {th }}$ AIAA Aerodynamic Decelerator Systems Technology Conference, Clearwater Beach, FL, May 15-18, 1995.

17. Development and Demonstration Test of a Ram-Air Parafoil Precision Guided Airdrop System, Volume 3, Simulation and Flight Test Results, Final Report CSDLR-2752, Draper Laboratory, U.S. Army Contract \#DAAK60-94-C-0041, October 1996.

18. Development and Demonstration Test of a Ram-Air Parafoil Precision Guided Airdrop System, Addendum: Flight Test Results and Software Updates after Flight 19, Final Report CSDL-R-2771, Draper Laboratory, U.S. Army Contract \#DAAK60-94-C-0041, December 1996.

19. Hattis, P.O., et al, "Precision Guided Airdrop System Flight Test Results," Proceedings of the $14^{\text {th }}$ AIAA Aerodynamic Decelerator Systems Technology Conference, San Francisco, CA, June 3-5, 1997.

20. Gockel, W., "Concept Studies of an Autonomous GNC System for Gliding Parachute," Proceedings of the $14^{\text {th }}$ AIAA Aerodynamic Decelerator Systems Technology Conference, San Francisco, CA, June 3-5, 1997.

21. Soppa, U., Strauch, H., Goerig, L., Belmont, J.P., and Cantinaud, O., "GNC Concept for Automated Landing of a Large Parafoil," Proceedings of the $14^{\text {th }}$ AIAA Aerodynamic Decelerator Systems Technology Conference, San Francisco, CA, June 3-5, 1997.
22. Jann, T., "Aerodynamic Model Identification and GNC Design for the Parafoil-Load System ALEX," AIAA2001-2015, Proceedings of the 16 ${ }^{\text {th }}$ AIAA Aerodynamic Decelerator Systems Technology Conference, Boston, MA, May 15-18, 2001.

23. Bryson, A.E. Dynamic Optimization, Addison Wesley Longman, Menlo Park, CA, 1999.

24. Gimadieva, T. Z.,"Optimal Control of a Guiding Gliding Parachute System," Journal of Mathematical Sciences, Vol.103 (1), 2001, pp.54-60.

25. Pontrjagin, L., Boltjanskiy, V., Gamkrelidze, R., and Mishenko, E. Mathematical Theory of Optimal Processes, Nayka, Moscow, 1969 (in Russian).

26. Moiseev, N.N., Elementi Teorii Optimal'nikh System (Elements of the Theory of Optimal Systems), Nauka, Moscow, 1975 (in Russian).

27. Tsai, H.B., "Analytical and Experimental Study of a Recovery Guidance System using Vortex Lattice Method, Linear Quadratic Controller and Proportional Navigation Guidance," M.S. Thesis, Iowa State University, 1999.

28. Tsai, H.B., Meiners, M.A., and Lahr, E.K., "Analytical and Experimental Study of a Recovery Guidance System at the Iowa State University, Iowa State University Publication, 1999.

29. Doherr, K.-F., and Jann, T., "Test Vehicle ALEX for Low-Cost Autonomous Parafoil Landing Experiments," AlAA-97-1543, Proceedings of the $14^{\text {th }}$ AIAA Aerodynamic Decelerator Systems Technology Conference, San Francisco, CA, June 3-5, 1997.

30. Jann, T., Doherr, K.-F., and Gockel, W., "Parafoil Test Vehicle ALEX- Further Development and Flight Test Results," AIAA-99-1751, Proceedings of the $3^{\text {rd }}$ AIAA Workshop on Weakly Ionized Gases, Norfolk, VA, November 1-5, 1999.

31. Petry, G., Hummeltenberg, G., and Tscharntke L., "The Parafoil Technology Demonstration Project," AIAA-971425 , Proceedings of the $14^{\text {th }}$ AIAA Aerodynamic Decelerator Systems Technology Conference, San Francisco, CA, June 3-5, 1997.

32. Klijn J.M., "The Instrumentation for the ESA Parafoil Technology Demonstrator Test," Proceedings of the $10^{\text {th }}$ SFTE European Chapter Symposium, Linkoping, Sweden, June 15-17 1998.

33. Petry, G., Behr, R., and Tscharntke, L., "The Parafoil Technology Demonstration (PTD) Project - Lessons Learned and Future Visions," AIAA-99-1755, Proceedings of the $15^{\text {th }}$ CEAS/AIAA Aerodynamic Decelerator Systems Technology Conference, Toulouse, France, June 8-11, 1999.

34. Kaminer, I., Pascoal, A., Hallberg, E., and Silvestre, C., "Trajectory Tracking for Autonomous Vehicles: An Integrated Approach to Guidance and Control," Journal of Guidance, Control, and Dynamics, vol.21 (1), 1998, pp.29-38.

35. Mortaloni, P., Yakimenko, O., Dobrokhodov, V., Howard, R., "On the Development of Six-Degree-of-Freedom Model of Low-Aspect-Ratio Parafoil Delivery System," Proceedings of $17^{\text {th }}$ AIAA Aerodynamic Decelerator Systems Technology Conference, Monterey, CA, May 1922, 2003. 
36. Costello, D., Kaminer, I., Carder, K., and Howard, R., "The Use of Unmanned Vehicle Systems for Coastal Ocean Surveys: Scenarios for Joint Underwater and Air
Vehicle Missions," Proceedings 1995 Workshop on Intelligent Control of Autonomous Vehicles, Lisbon, Portugal, 1995, pp. 61-72. 